\title{
Fragmented monopole crystal, dimer entropy, and Coulomb interactions in $\mathrm{Dy}_{2} \operatorname{Ir}_{2} \mathrm{O}_{7}$
}

\author{
V. Cathelin, ${ }^{1}$ E. Lefrançois, ${ }^{1,2}$ J. Robert, ${ }^{1}$ P. C. Guruciaga $\odot,{ }^{3}$ C. Paulsen, ${ }^{1}$ D. Prabhakaran, ${ }^{4}$ P. Lejay, ${ }^{1}$ F. Damay, ${ }^{5}$ J. Ollivier,${ }^{2}$ \\ B. Fåk, ${ }^{2}$ L. C. Chapon, ${ }^{6,2}$ R. Ballou $\odot,{ }^{1}$ V. Simonet, ${ }^{1}$ P. C. W. Holdsworth,,${ }^{7, *}$ and E. Lhotel ${ }^{1, \dagger}$ \\ ${ }^{1}$ Institut Néel, CNRS \& Université Grenoble Alpes, F-38042 Grenoble, France \\ ${ }^{2}$ Institut Laue Langevin, CS 20156, F-38042 Grenoble, France \\ ${ }^{3}$ Centro Atómico Bariloche, Comisión Nacional de Energía Atómica (CNEA), Consejo Nacional de Investigaciones Científicas y Técnicas \\ (CONICET), Avenida E. Bustillo 9500, R8402AGP San Carlos de Bariloche, Río Negro, Argentina \\ ${ }^{4}$ Clarendon Laboratory, Physics Department, Oxford University, Oxford OX1 3PU, United Kingdom \\ ${ }^{5}$ Laboratoire Léon Brillouin, Université Paris-Saclay, CNRS, CEA, CEA-Saclay, F-91191 Gif-sur-Yvette, France \\ ${ }^{6}$ Diamond Light Source Limited, Harwell Science and Innovation Campus, Didcot, United Kingdom \\ ${ }^{7}$ Université de Lyon, ENS de Lyon, Université Claude Bernard, CNRS, Laboratoire de Physique, F-69342 Lyon, France
}

(Received 1 October 2019; revised 1 September 2020; accepted 2 September 2020; published 29 September 2020)

\begin{abstract}
Neutron scattering, specific-heat, and magnetization measurements on both powders and single crystals reveal that $\mathrm{Dy}_{2} \mathrm{Ir}_{2} \mathrm{O}_{7}$ realizes the fragmented monopole crystal state in which antiferromagnetic order and a Coulomb phase spin liquid coinhabit. The measured residual entropy is that of a hard-core dimer liquid, as predicted. Inclusion of Coulomb interactions allows for a quantitative description of both the thermodynamic data and the magnetization dynamics, with the energy scale given by deconfined defects in the emergent ionic crystal. Our data reveal low-energy excitations, as well as a large distribution of energy barriers down to low temperatures, while the magnetic response to an applied field suggests that domain wall pinning is important, results that call for further theoretical modeling.
\end{abstract}

DOI: 10.1103/PhysRevResearch.2.032073

The fractionalization of microscopic elements into collective objects of reduced dimension has been a key concept in condensed matter for several decades [1]. In three dimensions the emergence, in frustrated pyrochlore magnets, of effective fields with $U(1)$ symmetry [2] provides an important, geometrically driven, and experimentally relevant source of fractionalization [3]. In particular, in spin ice materials [4] and models $[5,6]$, the associated topological charge, dressed by real magnetic flux, provides the magnetic monopole excitations [6,7] which have been much studied over the last decade. In this case, the magnetic moment configurations follow closely the emergent field theoretic picture and appear to fragment into two orthogonal fluids via a Helmholtz decomposition [8]. The two components act independently and in the right conditions can even order independently, giving the possibility of a monopole charge crystal [8-11], an antiferromagnetically ordered phase [12-15] which coexists with a ferromagnetically correlated Coulomb phase [16].

Pyrochlore iridates $R_{2} \mathrm{Ir}_{2} \mathrm{O}_{7}$, where the rare earth $R$ and iridium form interpenetrating pyrochlore structures, are ideal materials to generate such physics on the magnetic rare-earth

\footnotetext{
*Corresponding authors: peter.holdsworth@ens-lyon.fr

${ }^{\dagger}$ Corresponding authors: elsa.lhotel@ neel.cnrs.fr
}

Published by the American Physical Society under the terms of the Creative Commons Attribution 4.0 International license. Further distribution of this work must maintain attribution to the author(s) and the published article's title, journal citation, and DOI. sublattice. In these compounds, the $\mathrm{Ir}^{4+}$ sublattice orders magnetically into an "all-in-all-out" configuration (AIAO), with spins oriented along the local $\langle 111\rangle$ directions [17-20], at temperatures between 30 and $150 \mathrm{~K}$ [21] $\left(\operatorname{Pr}_{2} \operatorname{Ir}_{2} \mathrm{O}_{7}\right.$ being an exception). As the $R-R$ interactions are generally in the degrees Kelvin range, a good starting approximation is to treat this order as a staggered magnetic field which favors the same AIAO configurations for the rare-earth spins $[17,19]$. Within the monopole picture, this corresponds to a staggered chemical potential [11] which reduces the point group symmetry of the monopole sites and opens the door to the stabilization of the fragmented monopole crystal phase when $R-R$ interactions are ferromagnetic.

In this Rapid Communication, we show that $\mathrm{Dy}_{2} \mathrm{Ir}_{2} \mathrm{O}_{7}$ realizes such a fragmented monopole crystal state at temperatures below around $1 \mathrm{~K}$. We show that half the total moment of the $\mathrm{Dy}^{3+}$ ions is devoted to each of the magnetic sectors while specific-heat measurements expose the predicted residual entropy, which is that of a hard-core dimer fluid on the diamond lattice $[8,22,23]$. We model the results, including Coulomb interactions between monopoles [6], finding good qualitative agreement with experiment, with our analysis highlighting the role of long-range interactions for both static and dynamic measurements. However, our analysis also reveals the existence of low- and high-energy excitations that are not accounted for by simple models. Our results are compatible with previous experiments on $\mathrm{Ho}_{2} \mathrm{Ir}_{2} \mathrm{O}_{7}$ [12], but go considerably beyond them in presenting quantitative measures of both the Coulomb phase and the magnetic, ionic crystal. 


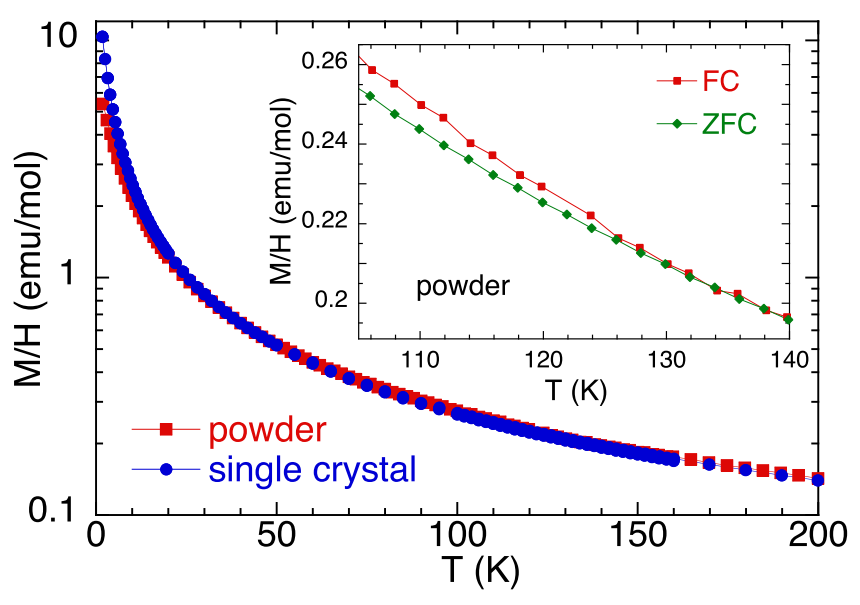

FIG. 1. FC magnetization $M / H$ vs temperature for the powder sample $(H=100 \mathrm{Oe})$ and a single crystal $(H=1000$ Oe applied in an arbitrary direction) on a semilogarithmic scale. Inset: Zoom in the ZFC-FC magnetization of the powder sample $(H=100 \mathrm{Oe})$.

Both polycrystalline and small single-crystal $\left(\sim 0.01 \mathrm{~mm}^{3}\right)$ samples were used [24]. Polycrystalline samples were characterized by neutron diffraction on the G4.1 (LLB) diffractometer down to $70 \mathrm{mK}$, and by inelastic neutron scattering down to $1.6 \mathrm{~K}$ on IN4 and IN6 (ILL) [24,25]. The latter measurements allowed us to refine the $\mathrm{Dy}^{3+}$ crystal electric field, giving an Ising ground-state doublet with a magnetic moment $m=$ $9.85 \mu_{\mathrm{B}}$ [24]. Magnetization measurements were performed down to $2 \mathrm{~K}$ on a Quantum Design (QD) magnetic property measurement system (MPMS) and a QD superconducting quantum interference device vibrating sample magnetometer (SQUID VSM), and between $90 \mathrm{mK}$ and $4 \mathrm{~K}$ on purpose-built SQUID magnetometers equipped with a miniature dilution refrigerator [26]. Specific-heat measurements were performed between 0.4 and $20 \mathrm{~K}$ with a ${ }^{3} \mathrm{He}$ QD physical property measurement system (PPMS) on the same single crystal (of mass $0.27 \mathrm{mg}$ ) as the QD SQUID VSM measurements. The specific heat of a pellet of $\mathrm{Eu}_{2} \mathrm{Ir}_{2} \mathrm{O}_{7}$ powder was measured as a reference nonmagnetic rare earth. For the very low-temperature SQUID measurements, several single crystals were coaligned.

As in other pyrochlore iridates [21], the iridium AIAO ordering manifests through a small irreversibility in the zero field cooled-field cooled (ZFC-FC) magnetization of the powder sample, below about $125 \mathrm{~K}$ (see Fig. 1), a slightly smaller temperature than the $134 \mathrm{~K}$ reported in Ref. [21]. The irreversibility is very small compared to this earlier study while no irreversibility could be detected for single crystals. The ZFC-FC irreversibility has been proposed to be due to structural defects and domain walls, which modify the iridium molecular field felt by the rare-earth ions, resulting in the enhancement of their polarization with decreasing temperature $[19,27,28]$. This scenario would suggest that our samples are cleaner than those used in previous reports.

Magnetic Bragg peaks appear in powder neutron diffraction measurements below about $100 \mathrm{~K}$ [see Fig. 2(a)]. FULLPROF refinements [29] with a $\mathbf{k}=\mathbf{0}$ propagation vector give an AIAO magnetic structure $[19,20,30]$ [shown in the inset of Fig. 2(b)] for both the Dy and Ir ions. The lowtemperature iridium ordered moment is found to be constant
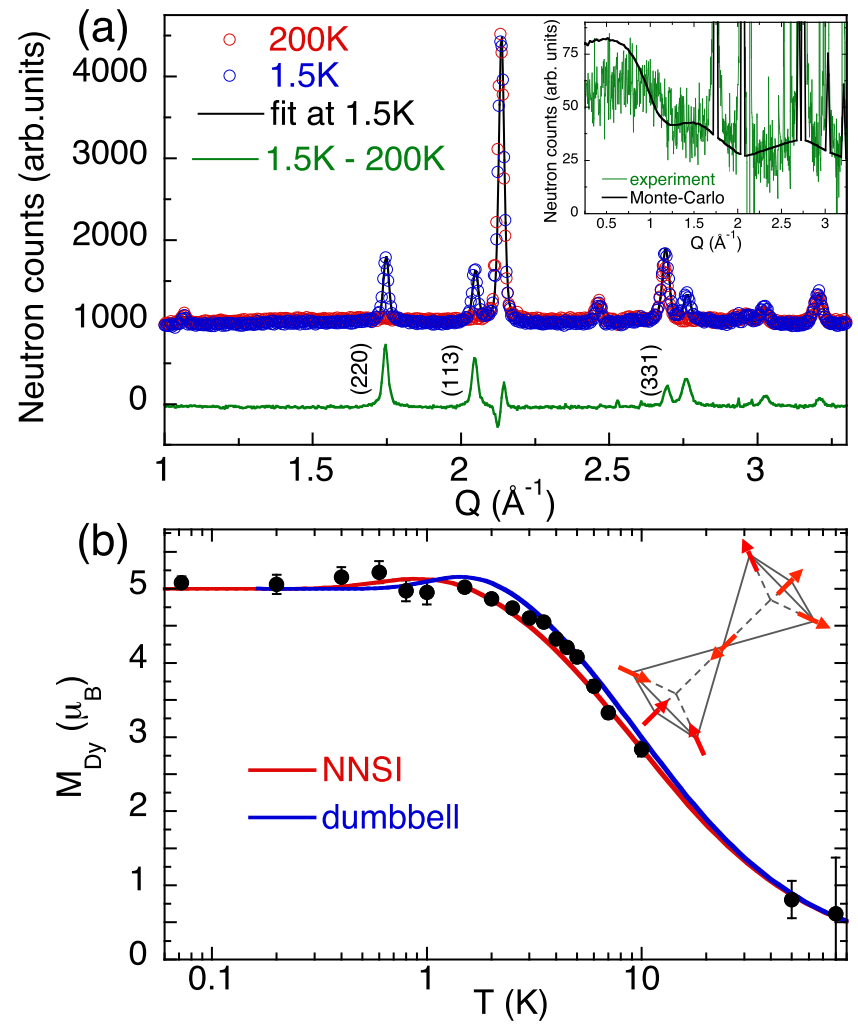

FIG. 2. (a) Diffractograms at $T=1.5$ (blue) and $200 \mathrm{~K}$ (red), and difference between 1.5 and $200 \mathrm{~K}$ (green). The black line is the refinement obtained at $1.5 \mathrm{~K}$. Inset: Zoom of the difference, corrected from the paramagnetic scattering (green). The black line is the powder average magnetic scattering function from Monte Carlo calculations in the nearest-neighbor spin ice (NNSI) model for $T / J_{\text {eff }}=0.05$ with $h_{\text {loc }} / J_{\text {eff }}=4.5$. (b) Refined Dy ${ }^{3+}$ ordered magnetic moment vs temperature between $80 \mathrm{~K}$ and $60 \mathrm{mK}$. Lines are the calculated ordered moment in the NNSI model for $\mathcal{J}_{\text {eff }}=1.1 \mathrm{~K}$ and $h_{\text {loc }} / \mathcal{J}_{\text {eff }}=4.5$ (red) and in the dumbbell model for $\mu=-4.40 \mathrm{~K}$ and $\Delta=4.95 \mathrm{~K}$ (blue). Inset: AIAO configuration on two tetrahedra.

in the analysis range $(T<80 \mathrm{~K})$ and equal to $m_{\mathrm{Ir}}=0.34 \pm$ $0.14 \mu_{\mathrm{B}}$. The temperature dependence of the ordered moment per $\mathrm{Dy}^{3+}$ ion $m_{\text {Dy }}$ between 10 and $80 \mathrm{~K}$ [Fig. 2(b)] is characteristic of field-induced order [19]. At lower temperature, Dy-Dy interactions favor a spin ice state, which competes with this field-induced state, leading to a saturation of $m_{\mathrm{Dy}}$ below $T=1.5 \mathrm{~K}$ to the value of $5 \pm 0.1 \mu_{\mathrm{B}}$, that is, to half of the total moment, as expected in the fragmentation scenario. Some diffuse magnetic signal persists down to the lowest temperature [see the inset of Fig. 2(a)]. These measurements thus provide two essential fingerprints for the stabilization of a fragmented crystal state in $\mathrm{Dy}_{2} \mathrm{Ir}_{2} \mathrm{O}_{7}$ : AIAO ordering accounting for half of the magnetic moment coexisting with a correlated spin-liquid phase.

As the local field lowers the symmetry of the monopole sites to that of the zinc-blende structure [31], a thermal phase transition is not required and none is observed in specific-heat measurements [see Fig. 3(a)]. However, as one enters fully into the fragmented phase a broad peak is observed, with a maximum at about $T=1.4 \mathrm{~K}$. This is reminiscent of the signal observed in classical spin ice [32] but is even broader, 

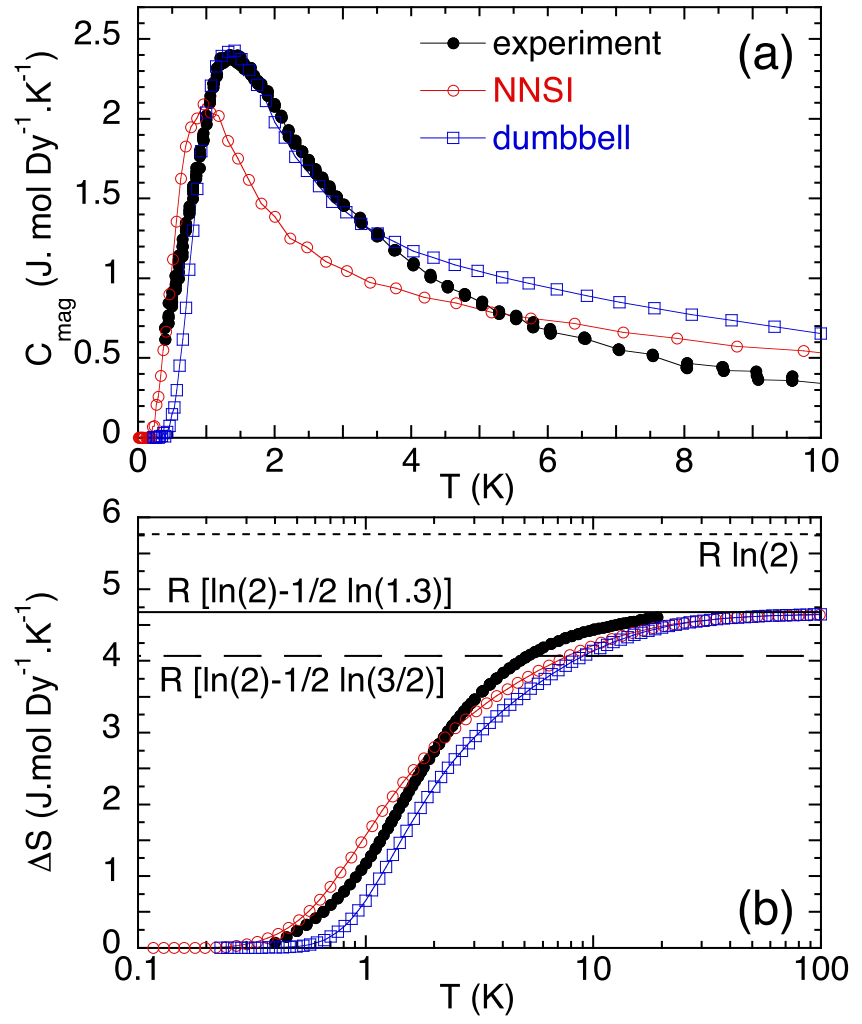

FIG. 3. (a) $C_{\text {mag }}$ vs $T$. The experiment (black dots) is compared to the NNSI (red circles, $h_{\text {loc }} / \mathcal{J}_{\text {eff }}=4.5$ and $\mathcal{J}_{\text {eff }}=1.1 \mathrm{~K}$ ) and the dumbbell (blue squares, $\mu=-4.40 \mathrm{~K}$ and $\Delta=4.95 \mathrm{~K}$ ) models. Specific-heat data of $\mathrm{Eu}_{2} \mathrm{Ir}_{2} \mathrm{O}_{7}$ with nonmagnetic $\mathrm{Eu}$ were subtracted from the original data to extract the Dy magnetic contribution [24]. (b) Entropy obtained from the integration of the above curves (semilogarithmic scale). $R \ln (2)$ corresponds to the full spin entropy, $R[\ln (2)-1 / 2 \ln (1.3)]$ to the fragmented entropy, and $R[\ln (2)-1 / 2 \ln (3 / 2)]$ to the Pauling entropy of ice.

spreading out to a much higher temperature, reflecting the energy scale of the local field.

Our low-temperature results differ from previous studies, which report a broad maximum at about $5 \mathrm{~K}$ in the susceptibility [33] or a sharp peak in the specific heat at $1.2 \mathrm{~K}$ [34]. Nevertheless, our measurements performed on both a powder and single crystals synthesized in different laboratories are consistent with each other. In addition, most of our observations can be accounted for by the model developed below.

We model the magnetic Dy-Ir interaction by a temperatureindependent mean-field term. This is most easily considered using the nearest-neighbor spin ice model (NNSI) [12],

$$
\mathcal{H}=\mathcal{J}_{\text {eff }} \sum_{\langle i, j\rangle} \sigma_{i} \sigma_{j}-h_{\mathrm{loc}} \sum_{i} \sigma_{i},
$$

where $\mathcal{J}_{\text {eff }}$ is an effective, ferromagnetic nearest-neighbor coupling, $\sigma_{i}=1(-1)$ is a reduced spin variable pointing in (out) of an up tetrahedron [35], and $h_{\text {loc }}$ is a staggered magnetic field coming from the iridium ions. However, the monopole approximation for spin ice, including long-range interactions, is captured by the dumbbell model $[6,36,37]$. Here, magnetic charge $Q_{i}$ sits at the vertices $i$ of the diamond lattice, dual to the pyrochlore lattice, and the spin Hamiltonian is replaced by

$$
\mathcal{H}_{\mathrm{db}}=\frac{u}{2} \sum_{i \neq j}\left(\frac{a}{r_{i j}}\right) \hat{n}_{i} \hat{n}_{j}-\mu \sum_{i} \hat{n}_{i}^{2}-\Delta \sum_{i=1, N_{0}}(-1)^{i} \hat{n}_{i},
$$

where $\hat{n}_{i}=Q_{i} / Q=0, \pm 1, \pm 2$ is a site occupation variable, $Q=2 \mathrm{~m} / \mathrm{a}$ the monopole charge, $u=\frac{\mu_{0} Q^{2}}{4 \pi a}=2.82 \mathrm{~K}$ the Coulomb energy scale, $\mu<0$ the chemical potential, and $N_{0}$ the number of tetrahedra [11]. The staggered chemical potential $\Delta$ replaces $h_{\text {loc }}$ giving an energy difference for monopole creation on the two sublattices of the diamond lattice [8]. Note that, although the field acts on a dipole and chemical potential on a monopole, when reduced to units of energy they are equal, $h_{\mathrm{loc}}=\Delta[11]$.

We have fitted the experimental results for the Dy ordered moment with data from the NNSI, with $\mathcal{J}_{\text {eff }}=1.1 \pm$ $0.1 \mathrm{~K}$ and $h_{\mathrm{loc}}=4.95 \pm 0.25 \mathrm{~K}$. For the dumbbell model, parameters were chosen to simultaneously reproduce both the magnetization and the specific heat, giving $\mu=-4.40 \pm$ $0.10 \mathrm{~K}$ and $\Delta=4.95 \pm 0.15 \mathrm{~K}$ [see Figs. 2(b) and 3(a)]. The values of $\mathcal{J}_{\text {eff }}$ and $\mu$ are close to the estimates for $\mathrm{Dy}_{2} \mathrm{Ti}_{2} \mathrm{O}_{7}$ [38]. The $h_{\text {loc }} / \mathcal{J}_{\text {eff }}$ ratio is the same as for $\mathrm{Ho}_{2} \mathrm{Ir}_{2} \mathrm{O}_{7}$ and these values place $\mathrm{Dy}_{2} \mathrm{Ir}_{2} \mathrm{O}_{7}$ deep in the predicted fragmented crystal phase at low temperature $[11,12]$.

The NNSI model fits the temperature dependence of $m_{\text {Dy }}$ quite accurately but in doing so gives a poor representation of the specific heat [see Fig. 3(a)], as was the case for $\mathrm{Dy}_{2} \mathrm{Ti}_{2} \mathrm{O}_{7}$ [38]. Introducing long-range interactions, the dumbbell model reproduces both $m_{\text {Dy }}$ and the specific-heat peak height and position, although the agreement is less convincing in the wings at high and low temperature. Above $4 \mathrm{~K}$ the model specific heat remains considerably higher than that of the experiment, indicating that correlations exist out to even higher temperatures. More surprisingly, while the model specific heat drops exponentially at small temperature, the experimental data appear to fall more slowly, retaining entropy down to lower temperatures. This indicates that low-energy excitations are present, which are not accounted for theoretically. These may originate from corrections to the dumbbell model which lift the degeneracy of the Coulomb phase, to structural defects, or to low-energy excitations in the iridium sector that are not accounted for.

In the monopole crystal phase it is predicted that the closed loops of virtual spin flips should induce a residual entropy equal to that of an ensemble of hard-core dimers on a diamond lattice, $S \approx \frac{1}{2} \ln (1.3)=0.131$ per spin [8,22,23] and the models have this ground-state entropy built into them. This is confirmed in Fig. 3(b) where we show the entropy recovered through integrating $\frac{C}{T}$ for both experiment and simulation. Experimentally, despite the apparent quantitative difference with the models we also recover this residual entropy to an excellent approximation.

The dynamics of the fragmented state can be probed with magnetization and ac susceptibility measurements. A freezing is observed when the system enters the fragmented crystal state, which manifests as a separation at $T=1.4 \mathrm{~K}$ between the ZFC and FC magnetizations measured when cooling from $4 \mathrm{~K}$ (see Fig. 4). Although the shape of the curve is slightly different for powders and single crystals, the ratio $M_{\mathrm{ZFC}} / M_{\mathrm{FC}}$ is the same in both cases and reaches about 0.2 at $80 \mathrm{mK}$ 


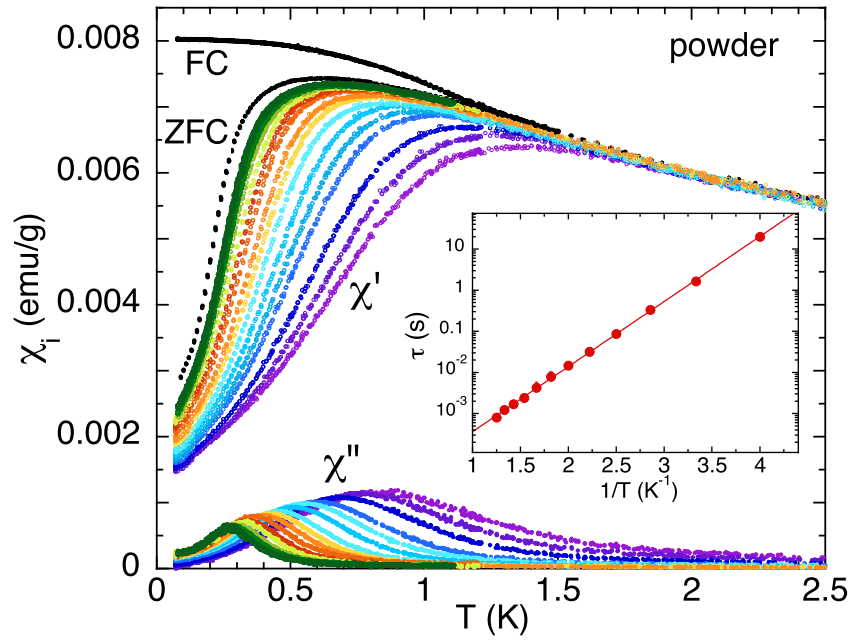

FIG. 4. ZFC-FC dc susceptibility (black points, $H=50 \mathrm{Oe}$ ) and ac susceptibility, $\chi^{\prime}$ and $\chi^{\prime \prime}$ (colored symbols, frequencies $f$ between 0.0057 and $211 \mathrm{~Hz}, H_{\mathrm{ac}}=1 \mathrm{Oe}$ ) vs temperature for the powder sample. Data were corrected for demagnetization effects with a demagnetization factor $N=0.1 \mathrm{cgs}$. Inset: Relaxation time $\tau=1 / 2 \pi f$ vs $1 / T$, obtained from the maximum of $\chi^{\prime \prime}$ vs $f$ measurements at fixed temperature [24]. The red line is a fit to the Arrhenius law $\tau=\tau_{0} \exp (E / T)$ with $\tau_{0}=9.4 \times 10^{-6} \mathrm{~s}$ and $E=3.6 \mathrm{~K}$.

[24]. The ZFC magnetization remains finite down to $80 \mathrm{mK}$, contrary to $\mathrm{Dy}_{2} \mathrm{Ti}_{2} \mathrm{O}_{7}$, where it falls to zero below $300 \mathrm{mK}$ [39]. This indicates that additional degrees of freedom exist that help magnetization to relax, consistently with our observations for the specific heat.

The ac susceptibility, shown in Fig. 4 exhibits a frequency dependence that can be accurately described by a thermally activated process, above an energy barrier $E=3.6 \mathrm{~K}$. This dynamics can be understood through the propagation of magnetically charged, deconfined defects in the monopole crystal [22]. In the dumbbell model, the lowest-energy excitation is a double monopole with energy [24]

$$
E_{\mathrm{db}}=-(3 \mu+\Delta)-u \alpha, \quad \alpha=1.638,
$$

which gives $E_{\mathrm{db}}=3.63 \mathrm{~K}$, in remarkable agreement with experiment. However, a word of caution is required; the propagation of the excitation, through a single spin flip, creates a hole of energy $E_{\mathrm{db}}^{\prime}=\mu+\Delta+u \alpha=5.2 \mathrm{~K}$. In order to avoid this higher-energy scale the dynamics would have to involve double spin flips $[3,22,24]$. The NNSI model underestimates these energies, giving $E_{\mathrm{NNSI}}=1.65$ and $2.75 \mathrm{~K}$, respectively [12], illustrating the importance of the Coulomb interaction between the magnetic charges. A Cole-Cole analysis of the ac susceptibility data shows that a large distribution of timescales exists, which broadens as the temperature decreases $[24,40,41]$. Just as for spin ice, this is compatible with quasiparticle hopping via a range of microscopic timescales [42-44].

The phase diagram of the fragmented crystal, as a function of applied magnetic field $\mathbf{H}$, is expected to be rich $[8,12,24]$. The magnetic field couples independently to the two fragmentation sectors, remarkably providing a staggered chemical potential for the monopoles $[6,11]$ in competition with the staggered internal field. As a consequence, a field placed

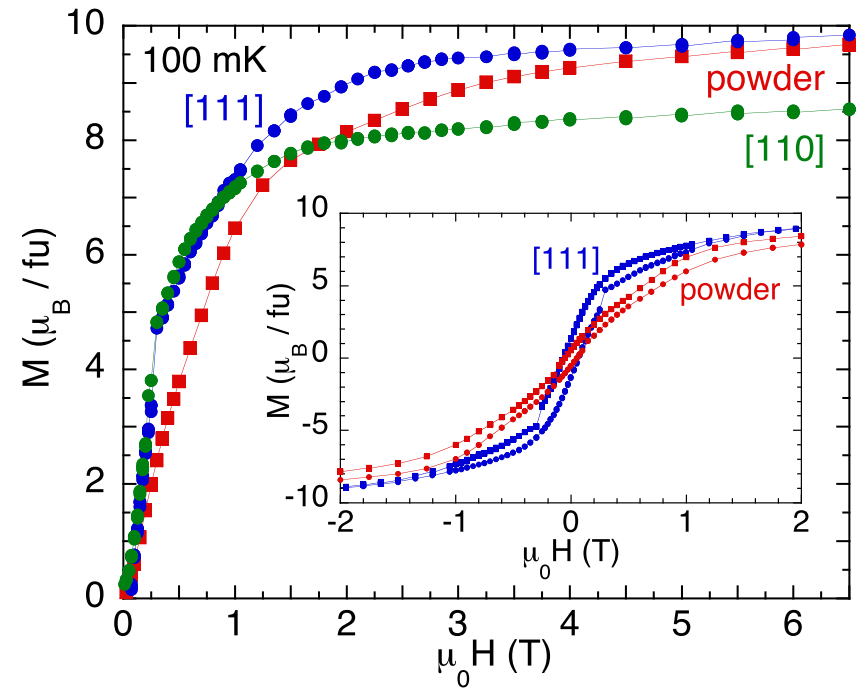

FIG. 5. Magnetization vs field at $T=100 \mathrm{mK}$ for the powder sample and the single crystal (H $\|$ [110] and [111]) measured starting from a ZFC state, except for the [111] direction where measurements were made from saturation in a negative field. Inset: Zoom in the hysteresis loop for the powder and $\mathbf{H} \|$ [111]. Curves have been symmetrized.

along the [111] (forward) and -[111] (reverse) directions is inequivalent, working with or against the internal field. In the forward direction the monopole configuration is unchanged by the field so that the magnetization should saturate via a Kasteleyn transition $[8,45]$ at low field. For $T=0.1 \mathrm{~K}$ the saturation field is only $6 \mathrm{mT}$ [24]. In the reverse direction the field generates a reduced effective $\Delta$, forcing the system back into the spin ice phase above a first threshold and into a monopole crystal going against the staggered field above a second threshold. This reorganization leads to three magnetization plateaus [12] and, for long-range interactions further phase transitions [11]. We predict a first plateau with $M=$ $m / 6$ for low field, jumping to a second at $m / 3$ for $\mu_{0} H \approx$ $1.3 \mathrm{~T}$, and to a third at saturation, for $\mu_{0} H \approx 3.2 \mathrm{~T}$ [24].

The availability of single crystals allows us to test these predictions. Data for fields placed along the [111] and [110] directions, together with measurements from powder samples, are shown in Fig. 5. The saturated magnetization per Dy approaches the expected values, $M_{[111], p w d}=m / 2$ and $M_{[110]}=$ $m / \sqrt{6}$ [46] for fields above $3 \mathrm{~T}$. The predicted magnetization plateaus are not observed, although the initial slope is steep, and in the powder data a change of slope is observed at around $1.5 \mathrm{~T}$, corresponding to the center of the second plateau.

An explanation for the absence of plateaus could be the presence of a partially frozen mosaic of "AIAO/AOAI" iridium domains (as observed in $\mathrm{Nd}_{2} \mathrm{Ir}_{2} \mathrm{O}_{7}$ [47]) which drive domains of monopole crystal order. In this case, the two kinds of domains would see the applied field as a forward or a reverse field. If completely frozen, the reverse domains would dominate the field response, resulting in the observation of plateaus for arbitrary magnetization values. Partial reorganization of the domain structure would result in a field-induced evolution of the fraction of the sample following the forward response scenario, masking the plateaus of the reverse 
response. Such a mixed response would terminate for fields around the upper threshold of $3.2 \mathrm{~T}$, which is consistent with the experimental results.

We observe a narrow hysteresis on field sweeping (see the inset of Fig. 5) which is consistent with the partial pinning of domains. It is accompanied in single crystals by small magnetization "avalanches," driven by self-heating as for $\mathrm{Dy}_{2} \mathrm{Ti}_{2} \mathrm{O}_{7}$ $[48,49]$, although the effect is less dramatic here, possibly due to the large thermal conductivity of the iridates or to additional relaxation channels offered by corrections to the simple models. The pinning appears stronger in the powder, where the remanence of the plateau is observed, which is consistent with our results at the iridium transition-see Fig. 1.

In conclusion, $\mathrm{Dy}_{2} \mathrm{Ir}_{2} \mathrm{O}_{7}$ stabilizes the fragmented monopole crystal state. Our analysis shows that both static and dynamic properties within this phase are governed by long-range interactions, captured in a first approximation by the monopole picture of spin ice. However, our measurements show evidence of low-energy excitations which are not generated by the model. Magnetization curves measured on single crystals do not show evidence of predicted magnetization plateaus, or of the reduced point group symmetry of the monopole crystal. This suggests that the role of the iridium has to be examined further, both at the microscopic level and in terms of its domain structure and dynamics.

We acknowledge A. Hadj-Azzem for his help in the compound synthesis, J. Debray for the orientation of the single crystals, and P. Lachkar for his help with the PPMS. V. Cathelin, C. Paulsen, P. C. W. Holdsworth and E. Lhotel acknowledge financial support from ANR, France, Grant No. ANR-15-CE30-0004. D. Prabhakaran acknowledges financial support from EPSRC, UK, Grant No. EP/N034872/1.
[1] P. Anderson, Science 235, 1196 (1987).

[2] S. V. Isakov, K. Gregor, R. Moessner, and S. L. Sondhi, Phys. Rev. Lett. 93, 167204 (2004).

[3] M. Hermele, M. P. A. Fisher, and L. Balents, Phys. Rev. B 69, 064404 (2004).

[4] M. J. Harris, S. T. Bramwell, D. F. McMorrow, T. Zeiske, and K. W. Godfrey, Phys. Rev. Lett. 79, 2554 (1997).

[5] B. C. den Hertog and M. J. P. Gingras, Phys. Rev. Lett. 84, 3430 (2000).

[6] C. Castelnovo, R. Moessner, and S. L. Sondhi, Nature (London) 451, 42 (2008).

[7] I. A. Ryzhkin, J. Exp. Theor. Phys. 101, 481 (2005).

[8] M. E. Brooks-Bartlett, S. T. Banks, L. D. C. Jaubert, A. Harman-Clarke, and P. C. W. Holdsworth, Phys. Rev. X 4, 011007 (2014).

[9] R. A. Borzi, D. Slobinsky, and S. A. Grigera, Phys. Rev. Lett. 111, 147204 (2013).

[10] P. C. Guruciaga, S. A. Grigera, and R. A. Borzi, Phys. Rev. B 90, 184423 (2014).

[11] V. Raban, C. T. Suen, L. Berthier, and P. C. W. Holdsworth, Phys. Rev. B 99, 224425 (2019).

[12] E. Lefrançois, V. Cathelin, E. Lhotel, J. Robert, P. Lejay, C. V. Colin, B. Canals, F. Damay, J. Ollivier, B. Fåk, L. C. Chapon, R. Ballou, and V. Simonet, Nat. Commun. 8, 209 (2017).

[13] S. Petit, E. Lhotel, B. Canals, M. Ciomaga Hatnean, J. Ollivier, H. Mutka, E. Ressouche, A. R. Wildes, M. R. Lees, and G. Balakrishnan, Nat. Phys. 12, 746 (2016).

[14] B. Canals, I.-A. Chioar, V.-D. Nguyen, M. Hehn, D. Lacour, F. Montaigne, A. Locatelli, T. O. Menteş, T. B. Santos Burgos, and N. Rougemaille, Nat. Commun. 7, 11446 (2016).

[15] J. A. M. Paddison, H. S. Ong, J. O. Hamp, P. Mukherjee, X. Bai, M. G. Tucker, N. P. Butch, C. Castelnovo, M. Mourigal, and S. E. Dutton, Nat. Commun. 7, 13842 (2016).

[16] C. L. Henley, Annu. Rev. Condens. Matter Phys. 1, 179 (2010).

[17] K. Tomiyasu, K. Matsuhira, K. Iwasa, M. Watahiki, S. Takagi, M. Wakeshima, Y. Hinatsu, M. Yokoyama, K. Ohoyama, and K. Yamada, J. Phys. Soc. Jpn. 81, 034709 (2012).
[18] H. Sagayama, D. Uematsu, T. Arima, K. Sugimoto, J. J. Ishikawa, E. O'Farrell, and S. Nakatsuji, Phys. Rev. B 87, 100403(R) (2013).

[19] E. Lefrançois, V. Simonet, R. Ballou, E. Lhotel, A. Hadj-Azzem, S. Kodjikian, P. Lejay, P. Manuel, D. Khalyavin, and L. C. Chapon, Phys. Rev. Lett. 114, 247202 (2015).

[20] H. Guo, C. Ritter, and A. C. Komarek, Phys. Rev. B 94, 161102(R) (2016).

[21] K. Matsuhira, M. Wakeshima, Y. Hinatsu, and S. Takagi, J. Phys. Soc. Jpn. 80, 094701 (2011).

[22] L. D. C. Jaubert, SPIN 5, 1540005 (2015).

[23] J. F. Nagle, Phys. Rev. 152, 190 (1966).

[24] See Supplemental Material at http://link.aps.org/supplemental/ 10.1103/PhysRevResearch.2.032073 for synthesis, crystal electric field (CEF), specific heat and extra magnetization data, Cole-Cole analysis, excitations, and magnetization in the dumbbell model.

[25] E. Lefrançois, R. Ballou, L. Chapon, B. Fåk, E. Lhotel, J Ollivier, and V. Simonet (2015), Magnetic and crystal field excitations in the pyrochlore iridates $R_{2} \operatorname{Ir}_{2} \mathrm{O}_{7}(R=\mathrm{Yb}, \mathrm{Dy}, \mathrm{Ho}$, Gd), Institut Laue-Langevin (ILL) doi:10.5291/ILL-DATA.401-1478.

[26] C. Paulsen, in Introduction to Physical Techniques in Molecular Magnetism: Structural and Macroscopic Techniques - Yesa 1999, edited by F. Palacio, E. Ressouche, and J. Schweizer (Servicio de Publicaciones de la Universidad de Zaragoza, Zaragoza, 2001), p. 1.

[27] W. C. Yang, W. K. Zhu, H. D. Zhou, L. Ling, E. S. Choi, M Lee, Y. Losovyj, C.-K. Lu, and S. X. Zhang, Phys. Rev. B 96, 094437 (2017).

[28] W. K. Zhu, M. Wang, B. Seradjeh, F. Yang, and S. X. Zhang, Phys. Rev. B 90, 054419 (2014).

[29] J. Rodriguez-Carvajal, Physica B 192, 55 (1993).

[30] H. Yan, O. Benton, L. Jaubert, and N. Shannon, Phys. Rev. B 95, 094422 (2017).

[31] D. E. Sands, Introduction to Crystallography (Dover, New York, 1969). 
[32] A. P. Ramirez, A. Hayashi, R. J. Cava, R. Siddharthan, and B. S. Shastry, Nature (London) 399, 333 (1999).

[33] K. Matsuhira, M. Wakeshima, Y. Hinatsu, C. Sekine, C. Paulsen, T. Sakakibara, and S. Takagi, J. Phys.: Conf. Ser. 320, 012050 (2011).

[34] D. Yanagishima and Y. Maeno, J. Phys. Soc. Jpn. 70, 2880 (2001).

[35] L. D. C. Jaubert and P. C. W. Holdsworth, J. Phys.: Condens. Matter 23, 164222 (2011).

[36] V. Kaiser, J. Bloxsom, L. Bovo, S. T. Bramwell, P. C. W. Holdsworth, and R. Moessner, Phys. Rev. B 98, 144413 (2018).

[37] C. Castelnovo and P. C. W. Holdsworth, in Spin Ice, edited by L. D. C. Jaubert and M. Udagawa (Springer, Berlin, 2019).

[38] R. G. Melko and M. J. P. Gingras, J. Phys.: Condens. Matter 16, R1277(R) (2004).

[39] J. Snyder, B. G. Ueland, J. S. Slusky, H. Karunadasa, R. J. Cava, and P. Schiffer, Phys. Rev. B 69, 064414 (2004).

[40] K. S. Cole and R. H. Cole, J. Chem. Phys. 9, 341 (1941).

[41] C. Dekker, A. F. M. Arts, H. W. de Wijn, A. J. van Duyneveldt, and J. A. Mydosh, Phys. Rev. B 40, 11243 (1989).
[42] L. D. C. Jaubert and P. C. W. Holdsworth, Nat. Phys. 5, 258 (2009).

[43] L. Bovo, J. Bloxsom, D. Prabhakaran, G. Aeppli, and S. T. Bramwell, Nat. Commun. 4, 1535 (2013).

[44] R. Dusad, F. K. K. Kirschner, J. C. Hoke, B. R. Roberts, A. Eyal, F. Flicker, G. M. Luke, S. J. Blundell, and J. C. S. Davis, Nature (London) 571, 234 (2019).

[45] R. Moessner and S. L. Sondhi, Phys. Rev. B 68, 064411 (2003).

[46] M. J. Harris, S. T. Bramwell, P. C. W. Holdsworth, and J. D. M. Champion, Phys. Rev. Lett. 81, 4496 (1998).

[47] E. Y. Ma, Y.-T. Cui, K. Ueda, S. Tang, K. Chen, N. Tamura, P. M. Wu, J. Fujioka, Y. Tokura, and Z.-X. Shen, Science 350, 538 (2015).

[48] D. Slobinsky, C. Castelnovo, R. A. Borzi, A. S. Gibbs, A. P. Mackenzie, R. Moessner, and S. A. Grigera, Phys. Rev. Lett. 105, 267205 (2010).

[49] M. J. Jackson, E. Lhotel, S. R. Giblin, S. T. Bramwell, D. Prabhakaran, K. Matsuhira, Z. Hiroi, Q. Yu, and C. Paulsen, Phys. Rev. B 90, 064427 (2014). 